Expositions : Le néo-réalisme italien au Fotomuseum de Winterthur|La photographie timbrée : l'inventivité visuelle de la carte postale photographique, Musée du Jeu de Paume (Paris), 4 mars-18 mai 2008

\title{
François Albera
}

\section{(2) OpenEdition}

\section{Édition électronique}

URL : https://journals.openedition.org/1895/2912

DOI : 10.4000/1895.2912

ISBN : 978-2-8218-0994-9

ISSN : 1960-6176

\section{Éditeur}

Association française de recherche sur l'histoire du cinéma (AFRHC)

\section{Édition imprimée}

Date de publication : 1 février 2008

Pagination : 179-187

ISBN : 978-2-913758-55-1

ISSN : 0769-0959

\section{Référence électronique}

François Albera, « Expositions : Le néo-réalisme italien au Fotomuseum de Winterthur | La photographie timbrée : l'inventivité visuelle de la carte postale photographique, Musée du Jeu de Paume (Paris), 4 mars-18 mai $2008 », 1895$. Mille huit cent quatre-vingt-quinze [En ligne], 54 | 2008, mis en ligne le 01 février 2011, consulté le 15 avril 2022. URL : http://journals.openedition.org/1895/2912 ; DOI : https:// doi.org/10.4000/1895.2912 


\section{Expositions}

\section{Le néo-réalisme italien}

\section{au Fotomuseum de}

\section{Winterthur}

II n'est sans doute pas besoin de rappeler ici ce qu'est le "néo-réalisme " au cinéma même si les délimitations chronologiques, thématiques, stylistiques et autres n'ont cessé de nourrir débats et controverses, en particulier en Italie, mais aussi en France. Débat dont on ne peut pas dire qu'il soit clos comme en témoignent les questions qui se posent encore à son propos dans les plus récents ouvrages italiens (tels ceux qu'a écrits ou dirigés Gian Piero Brunetta). L'expression, quoi qu'il en soit, a fait fortune et, comme il se doit, est devenu de plus en plus floue avec le temps. Elle s'appliquait déjà à la littérature de l'après-guerre, ici on l'applique à la photographie. Or cette dénomination, comme la période considérée, posent problème : pourquoi baptiser de la sorte après-coup et sur un laps de temps aussi long des photographes si différents au seul motif qu'ils ont saisi des images de la rue, des lieux de travail ou d'habitation, de préférence dans les régions déshéritées de la Péninsule, le Sud, la Sicile, la Calabre en particulier ? La dénomination en histoire de l'art n'est pas chose futile, elle est censée repérer un courant, un style commun voire des groupes et dès lors elle unifie, crée une cohérence qu'il faut parfois interroger sinon récuser pour y voir plus clair. Quand le seul le regard du critique ou du commissaire d'exposition (ici : Enricà Vigano) procède à cette opération on est en droit de s'interroger sur sa pertinence. Le " sujet "-gens du peuple, vie quotidienne, travail, misère - suffitil ? Un " style » ne comporte-t-il pas des partis pris esthétiques ? Et l'origine de la photo, sa destination ne comptent-ils pas autant que ce qui est photographié ? En 1935, Pasquale De Antonis photographie dans les Abruzzes, en cadrage penché, une petite fille au regard buté tenant devant elle une cage avec un perroquet; en 1950, Federico Patellani photographie en contre-plongée des mineurs en Sardaigne ; en 1958, Tranquillo Casiraghi photographie frontalement, sur leur pas de porte, des « gens » de Torretta ou du Pô. On pourrait en citer bien d'autres. Ce sont là leurs sujets mais que photographient-ils en eux ? Répondaient-ils à une commande d'État, d'une institution, d'un parti, d'un journal ? Voulaient-ils exalter, dénoncer, constater? Le mineur de charbon qui ressemble à Raf Vallone plonge 


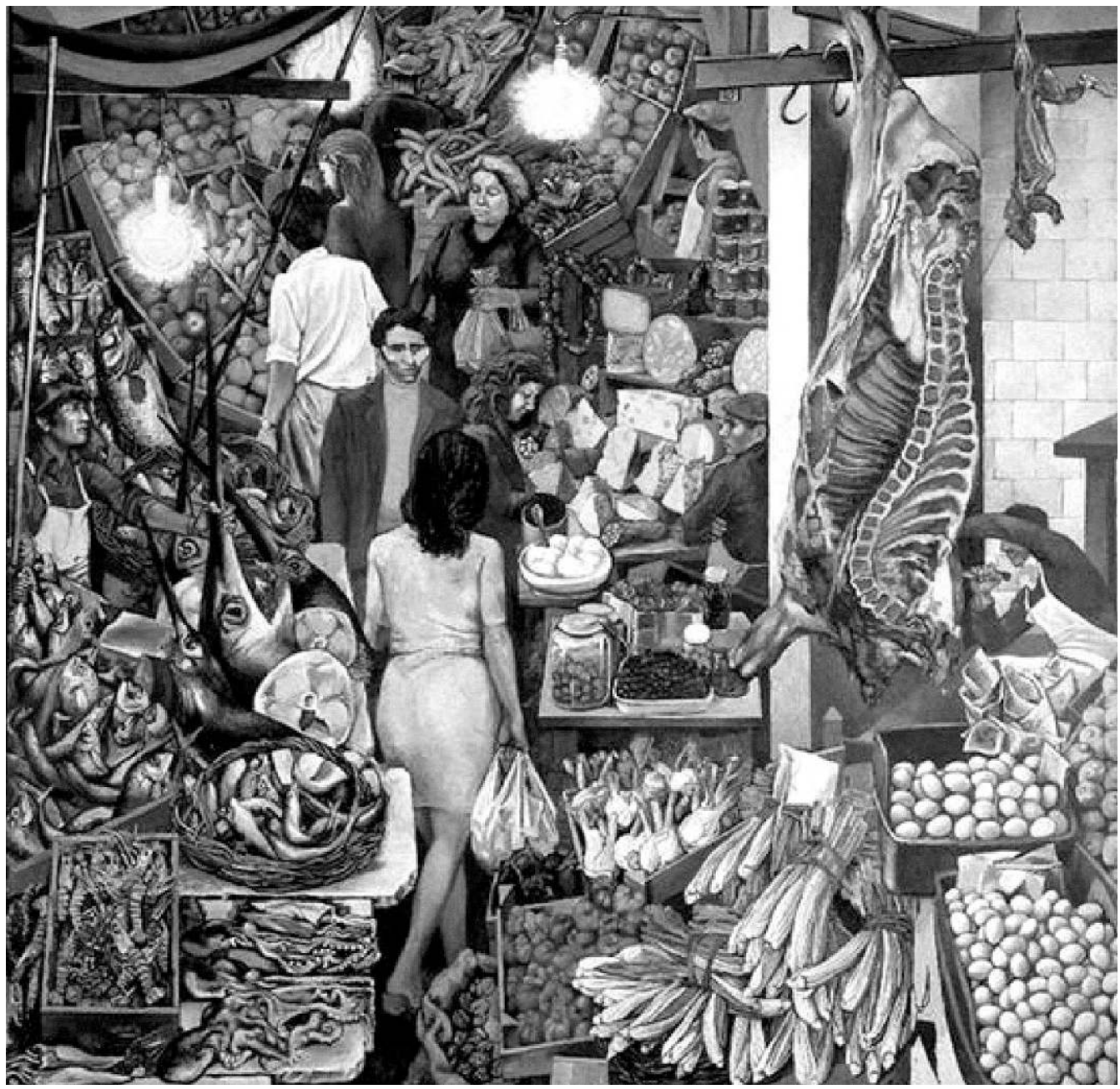

Renato Guttuso, Vucciria, Sicile, 1974

son regard dans l'objectif de l'appareil et donc l'œil du spectateur, le sourire de son compagnon, un peu en retrait, atténuant à peine sa détermination qui paraît celle d'un lutteur qui fait front. At-il simplement fini sa journée, est-il un gréviste, un « meneur »... On ne peut le savoir. Le pathos du cadrage penché sur la petite fille aux vêtements grossiers, ses yeux dans l'ombre en raison du contrejour, sa main burinée, abîmée par le travail agricole, qui avance vers nous et cet oiseau en cage, est-ce une allégorie de la misère, est-elle une mendiante ? 
Impossible de le savoir. Casiraghi reprenant le point de vue de la photo documentaire de Walker Evans est lui frontal, il élimine toute subjectivité du regard. Un mur lépreux, le trou sombre d'un intérieur, une vieille en noir assise, des bras comme des racines d'arbre, une femme plus jeune - sa fille ? - debout, bras croisés, vêtue de clair, souriante. Un sentiment de dignité, d'acceptation aussi de leur sort. Mais qu'y a-t-il au-delà de ces visages marqué par le soleil, ces corps marqués par le travail, ce dénuement ? On a rarement autant que dans cet ensemble disparates de photographies isolées le " besoin " de savoir ce qui " vient » avant et ce qui « viendra » après.
Quelques-unes appartiennent à des reportages et devraient donc être en série et liées à des textes, une mise en page (ainsi celles qui furent publiées dans Cinema nuovo, des années 1950) ou à une commande de l'Istituto Luce, actif sous le fascisme. D'autres sont le fruit d'une démarche individuelle : Giacomelli, qui inaugura vers 1958 un style âpre, contrasté minéralisant les visages de vieillards ou de malades mentaux par un travail au développement accentuant les noirs jusqu'à la saturation et faisant claquer les blancs, ne s'inscrit que de loin dans un éventuel courant social. Ugo Mulas encore moins, surtout connu pour ses photos d'artistes. On
$1895 /$

$n^{\circ} 54$

février 2008

181

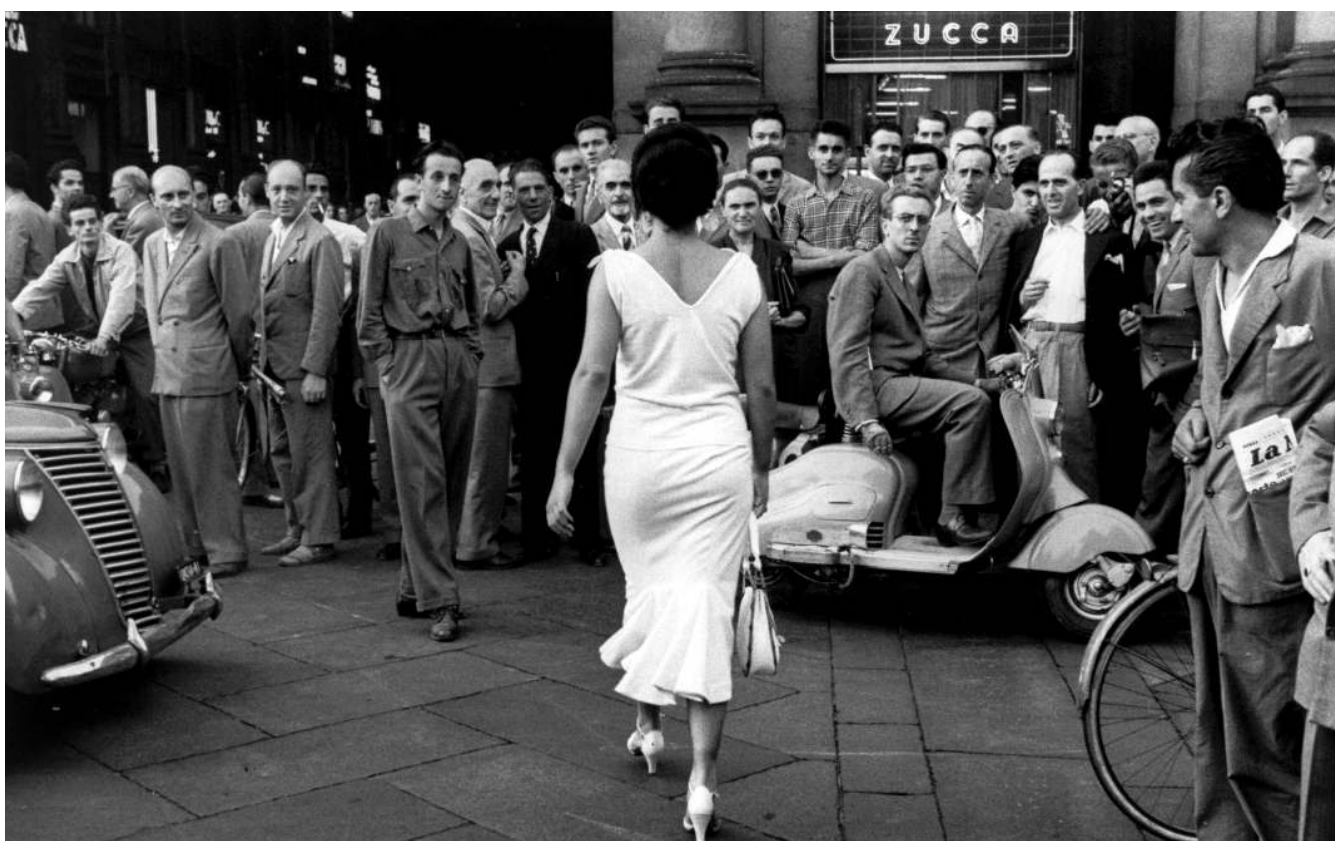




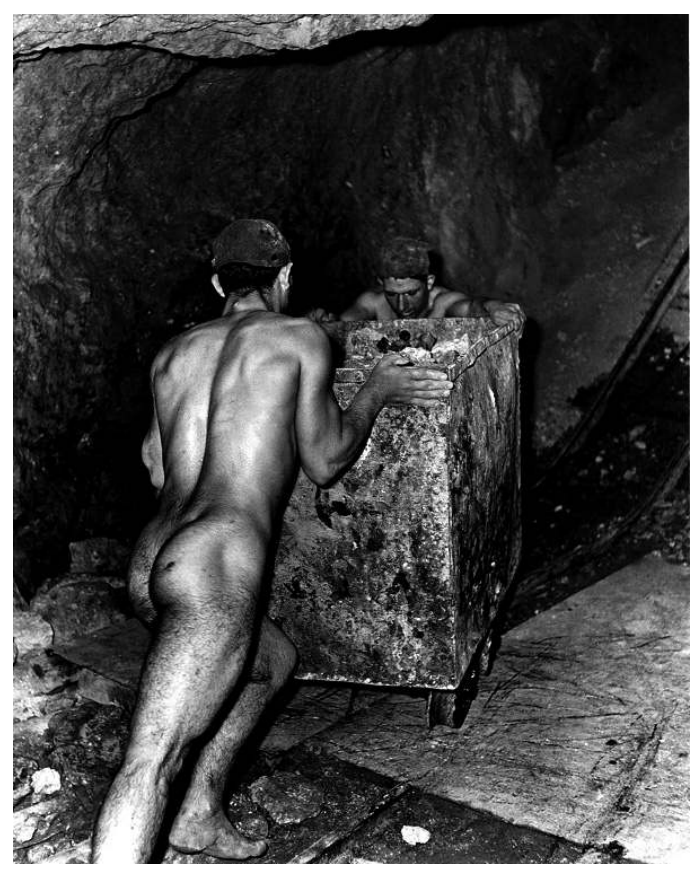

Federico Patellani, Mineurs de charbon, Sardaigne, 1950

montre ici quelques-unes de ses vues de Milan que I'on rapproche depuis longtemps par facilité de Miracolo a Milano de De Sica. Mais Alberto Lattuada, qui sera, lui, cinéaste dans le courant néoréaliste, engagé au sein du Parti communiste, est-il proche de ces deux-là ? Évidemment non. Les ambiguïtés d'une approche esthétique brouillent ainsi le sens de ces photos que leur " mise en scène » dans certains cas, rendent encore plus incertaines : ce mineur nu poussant un wagonnet de minerai, tant il fait chaud dans le sous-sol où il travaille, estil saisi là, comme il nous apparaît dans l'accrochage, pour la beauté sculpturale de ce corps luisant et sombre ou pour dénoncer ses conditions de travail ? Paradoxalement l'exposition esthétise le social et même la misère avec des images qui visaient à une documentation et souvent une dénonciation.

L'affiche de l'expo est un bon exemple de cette ambiguïté qui reprend une photo de Mario De Biasi réalisée à Milan en 1954. Une jeune femme toute en blanc, de dos, marche perpendiculairement à l'appareil vers une cinquantaine d'hommes arrêtés qui la regardent venir. Cent yeux dardés, interrogatifs, narquois, hilares, dubitatifs. Elle est manifestement une personnalité, une actrice peut-être, non une femme "quelconque ", et on est là dans une mise en scène, non dans la " vie à l'improviste " (on voit dans un coin un appareil photo). Il n'empêche que le poncif de I'Italien, de l'homme méditerranéen, du machisme fonctionne ici à bon marché. Il est intéressant de confronter cette image à la proposition que fit - sans aucun doute à partir d'elle - le peintre Renato Guttuso dans son Vucciria (1974). II répond au stéréotype de la femme " objet " et du regard prédateur des mâles en la plaçant dans l'allée d'un marché couvert, il en fait une ménagère avec son cabas en un lieu où rivalisent avec les formes féminines exaltées les 


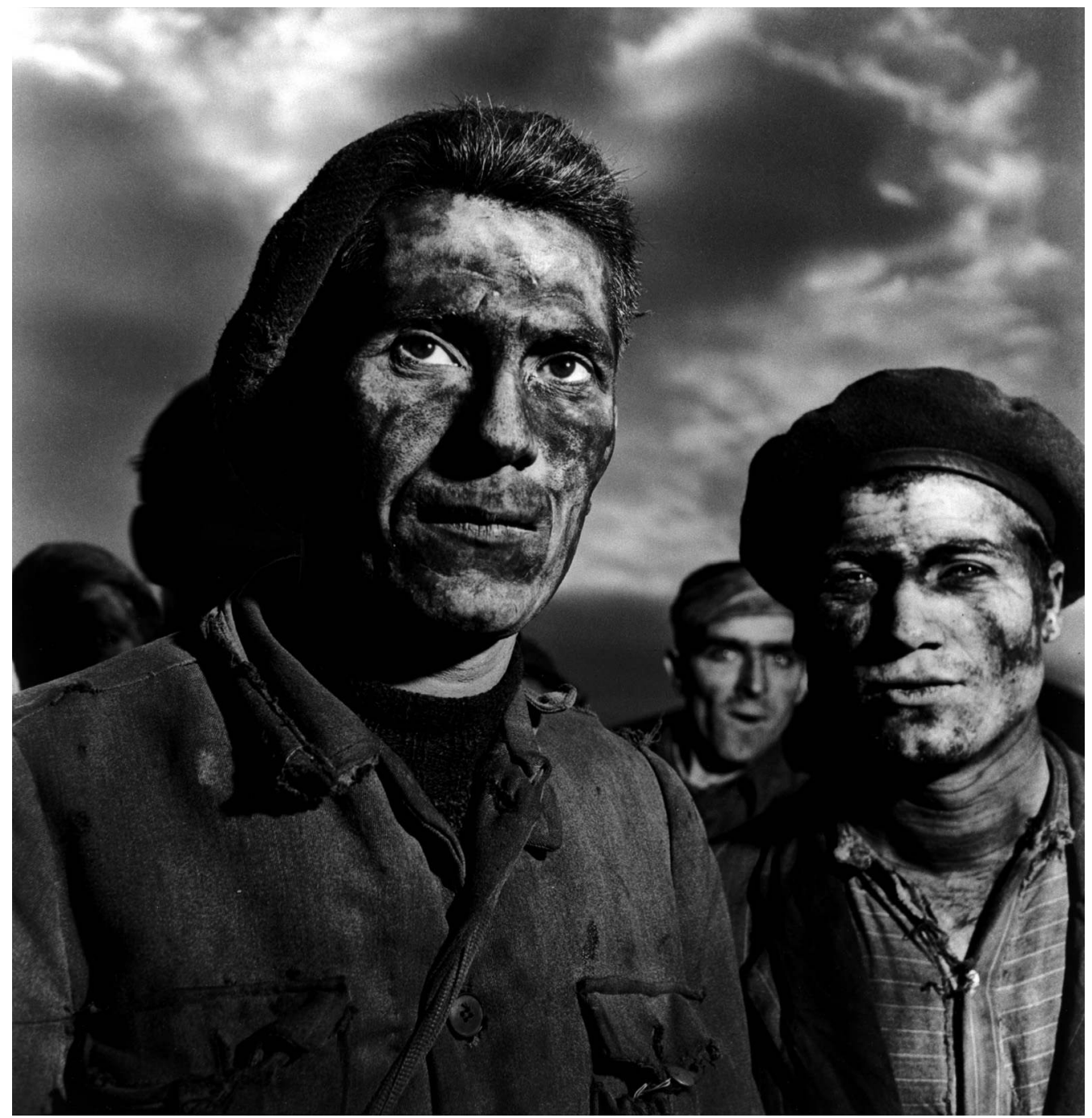

Fulvio Roiter, Dans une soufrière, Sicile, 1953] [facultatif]

étals de poissons, de légumes, de viandes et les gestes du travail du boucher découpant un quartier, du poissonnier qui a tranché la tête d'un thon, etc. Ce tableau est le lieu d'un retournement. Au lieu de diviser et d'opposer, il fait chanter une harmonie sensuelle des produits de la terre, de l'éros et du travail par la prolétarisation de la « star », inscrivant sur le visage d'un homme qui fait face une sorte d'absence, de flottement dû à la faim ou au désarroi. 
Malheureusement l'exposition ne pratique aucune réflexion de ce type ou d'un autre. Ce n'est certes pas la diffusion d'extraits de films sur des écrans video qui peut en tenir lieu. Aucun lien n'est établi et la qualité des extraits est, une fois de plus dans un cadre muséal, indigne : images grisâtres, mal définies et montrées en 16x9 qui étire et aplatit sans considérer le cadrage original.

II reste que cet élargissement d'une iconographie bien connue au cinéma atteste de la nécessaire contextualisation des films et, au-delà, des échanges et des intersections entre diverses pratiques de I'image. Dans un des premiers numéros des Cahiers du cinéma, Claude Roy publiait un reportage photographique sur les bidonvilles de Milan parallèlement à l'évocation du film de De Sica. On a relevé le fait que Cinema nuovo, la revue d'Aristarco, publiait des reportages photographiques sur la situation sociale. On pourrait encore citer l'intense réflexion de cette même revue engagea du côté des écrivains (Pavese, Fortini, Vittorini, Moravia notamment) pour évaluer la notion de " néo-réalisme » et s'interroger sur sa focalisation sur les pauvres et le sous-prolétariat en une sorte de mythologie sociale alors que l'enjeu politique se situait dans la classe moyenne.

François Albera
La photographie timbrée:

l'inventivité visuelle de la

carte postale photographique, Musée du Jeu de Paume (Paris), 4 mars18 mai 2008

Clément Chéroux mène depuis plusieurs années une recherche sur les " marges » de la photographie : cartes postales, trucages de foire, déformations, montages et collages. Il s'intéresse ici à l'un des supports de ces " déviances ", la carte postale. Née vers 1870, elle devient rapidement illustrée et par la photographie, grâce au progrès technique de la reproduction. L'engouement du public est considérable, des millions de cartes s'échangent saturant les services postaux et les collectionneurs se multiplient. Toute famille possède un ou plusieurs albums de ces cartes qui appartiennent à l'imagerie populaire (elles supplantent l'estampe). Comme l'écrit Chéroux, " les cartes postales photographiques constituent en somme la première alliance de deux modes de fascination, celui de l'image analogique et celui de l'image populai- 
re... C'est là, sans doute, la principale raison de leur très grand succès populaire ".

Souvent anonymes ces pratiques qui se développent autour de 1900 se retrouvent dans l'art d'avant-garde, dans les photomontages dadaïstes en particulier, puis dans le surréalisme qui ne cessera de leur donner une « légitimité » au nom du renversement des valeurs consacrées. S'agissant des cartes postales, le lien entre les deux est ici parfaitement illustré par Paul Eluard, poète surréaliste et grand collectionneur de cette imagerie amusante, insolite ; de même il ne manque pas de ces photos où le groupe de Breton, Aragon, Soupault, etc. se fait portraiturer en passant la tête dans des panneaux peints qui les transforment en aviateurs ou passagers d'une automobile. Ce lien entre les mouvements artistiques radicaux et ces fantaisies apparemment innocentes et bon enfant est évidemment ce qui excite l'intérêt : I'avant-garde récusant les valeurs, le sérieux académique ou la pose " artiste " repart du mot d'ordre de Rimbaud : "J'aimais les peintures idiotes ». Une manière d'être " mal élevé » qui révèle à la fois la dimension fantastique de l'imagerie de masse (on transforme les proportions, colle une petite tête sur un gros corps, une tête de cheval sur le corps du cocher, on fait se gondoler la Tour Eiffel, etc.) et les libertés qu'elle prend avec les conventions de la repré- sentation, le réalisme. L'« appropriation " qu'ils pratiquent, c'est-à-dire «l'intégration, dans l'œuvre d'un artiste, d'éléments traditionnellement considérés comme exogènes à la sphère de l'art ", concerne aussi bien la publicité, les logos des marques quand il ne s'agit pas simplement d'objets quotidiens (coupures de journaux, fragments de photographies, morceaux de tissu, tickets, bouts de bois, etc.,). Comme le dit très bien Chéroux, ce qui intéresse les artistes d'avant-garde dans cette imagerie, c'est son caractère anti-artistique et collectif. Dalì dira qu'ils y trouvaient l'expression d'une sorte d'inconscient collectif. Cependant la " reprise " avant-gardiste vise moins à amuser qu'à démonter, détruire ou en tout cas remettre en question un ordre représentatif garant de l'ordre social. La dimension subversive ne fait pas que "s'ajouter ", elle déplace les enjeux de ces pratiques. Les collages de Hannah Hoch, Raoul Haussmann, Hans Arp ou Georges Hugnet poussent les conséquences de ces « jeux " jusqu'au malaise, au trouble (notamment sur le plan du désir ou de la répulsion). D'ailleurs la démarche est similaire quand Max Ernst travaille avec des gravures populaires qu'il détourne (Une semaine de bonté, la Femme cent têtes) ou Duchamp avec des objets usuels. Au cinéma on peut songer à la reprise de motifs comiques ou mélodra- 


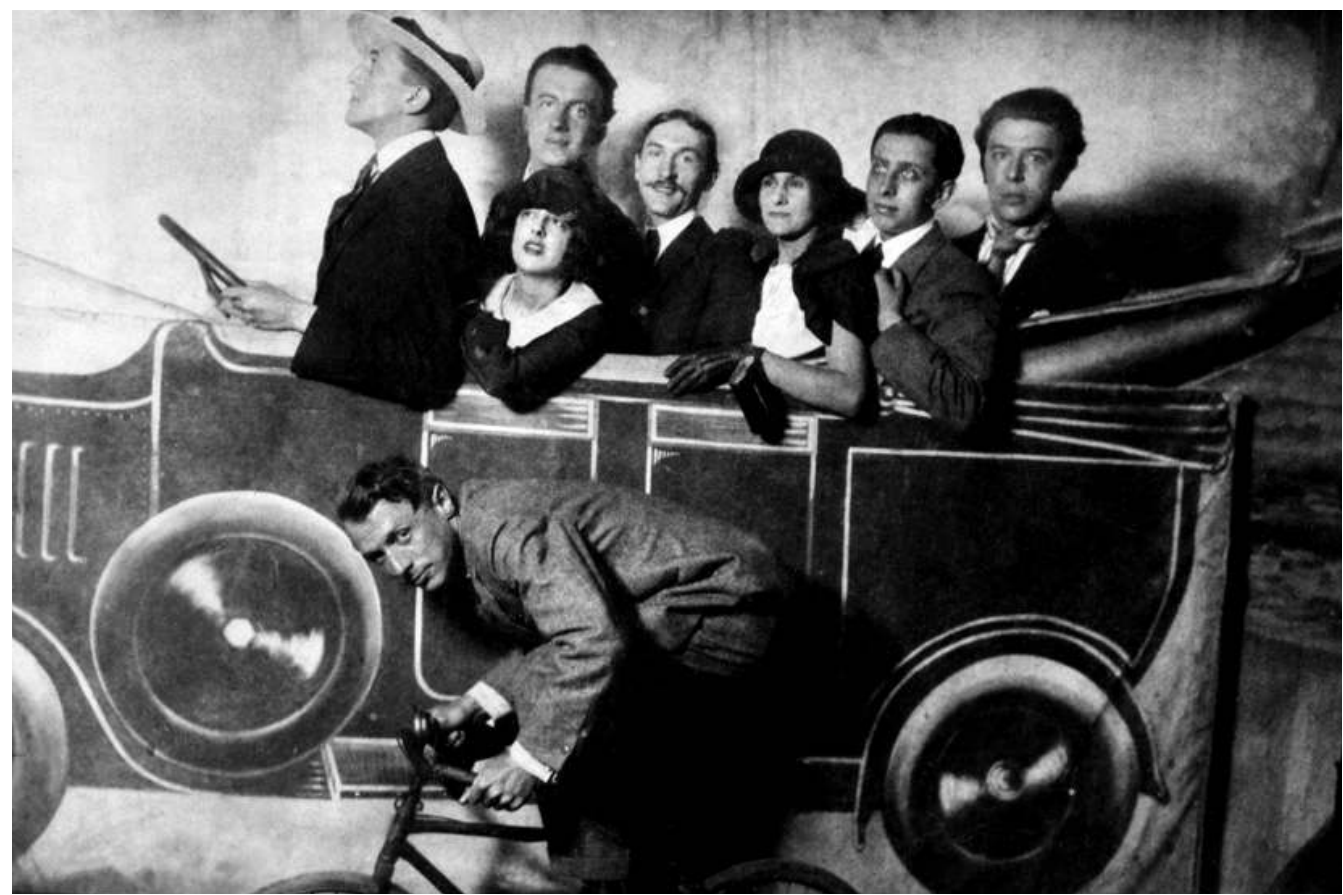

Max Morice, Max Ernst, Simone Breton, Paul Eluard, Joseph Delteil, Gala, Robert Desnos, André Breton en 1923 (studio de photographie inconnu)

matiques par Buñuel dans ses premiers films. Les fantaisies de cartes postales (qui se perpétuent d'ailleurs de nos jours - en général dans l'obscène) comme les caricatures des Incohérents et des Zutistes, comme les jouets optiques à transformation ou les films de Méliès ou des comiques "primitifs " demeurent respectueux des usages. Chacun reste à sa place. La force des dadaïstes est de ne pas respecter cet ordre des choses... Entre les deux, en quelque sorte, il y a le photomontage politique (absent de l'exposition : Heartfield) et la publicité ou la propagande (une série de Klutsis sur les
Spartakiades en URSS) où I'énergie déployée par cet insolite se retrouve canalisée dans une fin bien précise.

Ce qui frappe dans cette exposition comme dans les travaux et publications antérieures de Chéroux, c'est le territoire commun à la photographie et au cinéma qui, recourant pourtant à des procédés différents, recherchent les mêmes effets. L'Homme à la tête de caoutchouc de Méliès, obtenu par surimpressions, cache-contre cache, arrêt de caméra, etc. appartient à la même inspiration que cette imagerie anonyme, comme les manipulations graphiques ou les trucs 
d'Émile Cohl. Sadoul (pourtant collectionneur de cartes postales - Eluard est prêt à en échanger 200 contre un tableau de Dalì !) note en passant ces apparentements mais on voit bien, grâce à une exposition comme celle-ci, qu'il $s^{\prime}$ agit d'explorer de plus près la question et, sans doute, pour cela, envisager un domaine de l'image qui ne soit plus compartimenté en fonction des médiums respectifs.

Les choix de l'accrochage, de l'exposition proprement dite, établissent certains ponts avec d'autres pratiques iconiques ou artistiques, en particulier en s'efforçant de "dépasser " le petit format des cartes $(9 \times 14 \mathrm{~cm})$ peu propice à la mise en évidence. Ainsi en projetant et en agrandissant ces cartes, on va, en quelque sorte, à la rencontre de l'image filmique. En les diffusant sur un moniteur de télévision, on leur donne un autre statut médiatique. Enfin l'accumulation de cartes postales sur une surface à la manière des œuvres d'Annette Messager les relie à l'art contemporain.

François Albera

\section{De quoi Sacha Guitry}

\section{est-il le nom ?}

L'événement "Sacha Guitry » auquel on a été convié récemment - rétrospective, exposition, catalogue, réédition et édition - vient rejouer à l'identique de précédentes célébrations de "I'đFuvreet-I'Homme ", régulières malgré tout depuis la réévaluation qu'entreprit Truffaut de son cinéma (si l'on suit sur ce point ce que dit le catalogue de l'exposition). Il me souvient d'une rétrospective à Locarno, il y a quinze ans (première année de la direction de Marco Muller), reprise à la Cinémathèque française (alors dirigée par Païni) - assortie elle aussi d'un livre (chez Yellow Now) dont on disait qu'il ferait date. II serait sans doute intéressant (sujet de mémoire peut-être) de suivre au plus près les relais, les rebonds, la continuité de cette célébration, ce qui, en elle relève de la répétition. Et d'établir comment le cinéma et la biographie " sauvent " Guitry car on n'observe guère dans la critique littéraire ou théâtrale un semblable intérêt (même si on joue et on lit Guitry).

Il y aurait certainement plus à dire du combat de Truffaut, dont Rohmer emboîte le pas (et auquel on parvient, ouf !, à rallier Bazin du bout des lèvres), que ce qu'en écrit le catalogue. Celui-ci insiste néanmoins très justement sur la 